\title{
APPLICATION OF THE AVERAGING METHOD TO THE PROBLEMS OF OPTIMAL CONTROL OF THE IMPULSE SYSTEMS
}

\author{
KOVAL'CHUK T.V. ${ }^{1}$, MOgYlOVA V.V. ${ }^{2}$, STANZHYTSKYI O.M. ${ }^{3}$, SHOVKOPLYAS T.V. ${ }^{3}$
}

The problem of optimal control at finite time interval for a system of differential equations with impulse action at fixed moments of time as well as the corresponding averaged system of ordinary differential equations are considered.

It is proved the existence of optimal control of exact and averaged problems. Also, it is established that optimal control of averaged problem realize the approximate optimal synthesis of exact problem. The main result of the article is a theorem, where it is proved that optimal contol of an averaged problem is almost optimal for exact problem. Substantiation of proximity of solutions of exact and averaged problems is obtained.

Key words and phrases: optimal control problem, optimal control, exact problem, averaged problem, valid control, averaging condition, weak convergence, compactness, semi-continuity, quality criterion, minimizing sequence.

\footnotetext{
${ }^{1}$ Kyiv National University of Trade and Economics, 19 Kioto str., 02156, Kyiv, Ukraine

2 National Technical University of Ukraine "Igor Sikorsky Kyiv Polytechnic Institute", 7 Peremogy av., 03056, Kyiv, Ukraine

3 Taras Shevchenko National University of Kyiv, 64/13 Volodymyrska str., 01601, Kyiv, Ukraine

E-mail: optimalcontroleukr.net (Koval'chuk T.V.), ostanzh@gmail.com (Mogylova V.V.), ostanzh@gmail.com (Stanzhytskyi O.M.), optimalcontrol@ukr.net (Shovkoplyas T.V.)
}

\section{INTRODUCTION}

In this paper, for the system of differential equations with impulse action at fixed moments of time, the problem of optimal control is considered:

$$
\begin{gathered}
\dot{x}=\varepsilon[A(t, x)+B(t, x) u], \quad t \neq t_{i}, \quad i=1,2, \ldots, i\left(\frac{T}{\varepsilon}\right), \quad t \in\left[0, \frac{T}{\varepsilon}\right), \\
\left.\triangle x\right|_{t=t_{i}}=\varepsilon I_{i}\left(x\left(t_{i}\right), v_{i}\right), \quad i=1,2, \ldots, i\left(\frac{T}{\varepsilon}\right), \\
x\left(0, u(0), v_{i}\right)=x_{0}, \quad t_{i}<t_{i+1},
\end{gathered}
$$

where $\varepsilon>0$ is a small parameter, $t \geq 0, T>0$ is some constant value, $x \in D$ is a phase $n$ dimensional vector, $D$ is a region in $R^{n}, u \in U$ is a vector of control, $U$ is convex and closed set in $R^{m}, 0 \in U, i(t)$ is the number of pulses on $[0, t): t_{1}, t_{2}, \ldots, t_{n}, \ldots, t_{i\left(\frac{T}{\varepsilon}\right)}$, and $t_{n} \rightarrow \infty, n \rightarrow \infty$; $v_{i} \in V, i=1,2, \ldots, i\left(\frac{T}{\varepsilon}\right)$, are impulse control vectors, $V$ is a closed set in $\mathbb{R}^{r}$.

With respect to the moments of impulsive action, we assume that there exists a constant $\widetilde{C}>0$ such that for $t \geq 0$,

$$
i(t) \leq \widetilde{C} t
$$

$\mathrm{y} \Delta \mathrm{K} 517.9$

2010 Mathematics Subject Classification:34K35, 34N99, 39A12. 
Let $A$ be an $n$-dimensional vector-function, $B$ be an $n \times m$-dimensional matrix, $I_{i}(x, v)$ is an $n$-dimensional vector function. Control $u=u(t)=\left(u_{1}(t), u_{2}(t), \ldots, u_{m}(t)\right)$ and $v=v_{i}=$ $\left(v_{i 1}, v_{i 2}, \ldots, v_{i r}\right)$ will be considered admissible for problem $(1)$, if

a1) $u(t) \in L_{p}\left(0, \frac{T}{\varepsilon}\right)$ for some $p>1$;

a2) $u(t) \in U$ at $t \in\left[0, \frac{T}{\varepsilon}\right]$ almost everywhere;

a3) there exists $\varepsilon_{0}>0$ such that for $0<\varepsilon<\varepsilon_{0}$ the solution $x(t, u, v)$ of the Cauchy problem (1) has defined by $t \in\left[0, \frac{T}{\varepsilon}\right]$, where $\varepsilon_{0}$ is independent of $u(t)$ and $v_{i}$;

a4) $v_{i} \in V$;

a5) for each sequence of control vectors $v_{i} \in V$ there exists a vector $v_{0} \in V$ such that $v_{i} \rightarrow v_{0}$, $i \rightarrow \infty$, uniformly for all controls, that is, for arbitrary $\delta>0$ there is a constant $N_{0}$, independent of $v_{i}, v_{0}$ and such that for all $i \geq N_{0}$ the inequality $\left|v_{i}-v_{0}\right|<\delta$ is satisfied.

It should be noted that condition a5) is obviously satisfied if there exists a sequence $\left\{a_{i}\right\}$ independent of $v_{i}: a_{i} \rightarrow 0, i \rightarrow \infty$, such that $\left|v_{i}-v_{0}\right|<a_{i}$.

We denote the set of valid controls as $\Omega$. Though $|\cdot|$ we denote the norm of vector in Euclidean space, and through $\|\cdot\|$ we denote the norm of the matrix consistent with the norm of the vector.

In this paper, the averaging method is applied to optimal control problems. The main role here is to justify the closeness of the solutions of the exact and average problems. This type of results for impulse systems was obtained first in [4] and further developed in the works of many scientists and applied to optimal control problems (see, for example, [9], where there is a comprehensive bibliography).

In works [3,6-8], another approach was developed to apply the averaging method to optimal control problems, where the control function was considered a fixed parameter when averaging. This approach had applied to the problems of optimal control of functional-differential equations in [2].

This work continues the study of [1], however, unlike the work [1], the existence of optimal controls is also established here for the problem under consideration.

The structure of the article is as follows: the introduction discusses the problem is considering and provides a literature review, Section 1 gives a rigorous statement of the problem and formulates the main result, Section 2 formulates and proves the Lemma about averaging, Section 3 deals with proving of the main result.

\section{PROBlem STATEMENT AND FORMULATION OF THE MAIN RESUlTS}

The problem of optimal control to be solved in the work is to find such allowable controls $u(t)$ and $v_{i}$ that minimize the functional

$$
J_{\mathcal{\varepsilon}}(u, v)=\varepsilon \int_{0}^{\frac{T}{\varepsilon}}[C(t, x)+F(t, u)] d t+\varepsilon \sum_{0 \leq t_{i}<\frac{T}{\varepsilon}} \Psi_{i}\left(x\left(t_{i}\right), v_{i}\right),
$$

here $C, F, \psi_{i}$ are jointly continuous functions, where $C \geq 0, F$ and $\psi_{i}$ satisfy the conditions: 
$F(t, u)$ is defined for $t \geq 0, u \in U$, it is convex on $u$, and for some $a>0$

$$
F(t, u) \geq a|u|^{p}, \quad \psi_{i}(t, v) \geq a|v|^{p},
$$

where $p>1$ is as in condition $a 1)$ and for some $K>0$ there exists $\varepsilon_{0}>0$ such that for $\varepsilon<\varepsilon_{0}$ the inequality holds

$$
\varepsilon \int_{0}^{\frac{T}{\varepsilon}} F(t, 0) d t \leq K
$$

We assume that for system (1) the following conditions are fulfilled:

1.1) there are such $A_{0}(x), B_{0}(x)$ and $C_{0}(x)$, such that the following boundaries exist uniformly on $x \in D$ (averaging conditions):

$$
\begin{aligned}
& \lim _{T \rightarrow \infty}\left|\frac{1}{T} \int_{0}^{T} A(t, x) d t-A_{0}(x)\right|=0, \\
& \lim _{T \rightarrow \infty}\left|\frac{1}{T} \int_{0}^{T} C(t, x) d t-C_{0}(x)\right|=0, \\
& \lim _{T \rightarrow \infty} \frac{1}{T} \int_{0}^{T}\left\|B(t, x)-B_{0}(x)\right\|^{q} d t=0,
\end{aligned}
$$

where $q$ is determined from the condition $\frac{1}{p}+\frac{1}{q}=1$;

1.2) the vector function $A(t, x)$ and the matrix function $B(t, x)$ are measurable on $t$ for each $x$, the function $C(t, x)$ is continuous on $t \geq 0, x \in D$;

1.3) the functions $A(t, x), B(t, x)$ and $C(t, x)$ are Lipschitz's functions on $x$ with constant $L$ in domain $D$;

1.4) the functions $I_{i}(x, v), \psi_{i}(x, v), i=1,2, \ldots, i(t)$, are jointly continuous;

1.5) the functions $\psi_{i}(x, v), i=1,2, \ldots, i(t)$, are bounded by the constant $M$ at $t \geq 0, x \in D$, $v \in V$

1.6) the functions $I_{i}(x, v), \psi_{i}(x, v), i=1,2, \ldots, i(t)$, are Lipschitz's functions on $x$ with constant $L$ in the domain $D$ and uniformly continuous on $v$ in their domains;

1.7) for the functions $A(t, x), B(t, x), C(t, x)$ and $I_{i}(x, v), i=1,2, \ldots, i\left(\frac{T}{\varepsilon}\right)$, the conditions of linear growth are fulfilled, i.e., there is a constant $K>0$ such that for $t \geq 0$ and $x \in D$ the followings inequalities are fulfilled:

$$
\begin{array}{ll}
|A(t, x)| \leq K(1+|x|), & \|B(t, x)\| \leq K(1+|x|), \\
\left|I_{i}\right| \leq K(1+|x|), & |C(t, x)| \leq K(1+|x|) .
\end{array}
$$

Let the averaging conditions also be satisfied:

1.8) there exist the following limits uniformly on $x \in D, u \in U, v \in V$ :

$$
\lim _{s \rightarrow \infty} \frac{1}{s} \sum_{0<t_{i}<s} I_{i}(x, v)=I_{0}(x, v), \quad \lim _{s \rightarrow \infty} \frac{1}{s} \sum_{0<t_{i}<s} \psi_{i}(x, v)=\psi_{0}(x, v) .
$$


To the problem (1) on the interval $\left[0, \frac{T}{\varepsilon}\right]$ we will correspond the following averaged problem:

$$
\begin{gathered}
\dot{y}=\varepsilon\left[A_{0}(y)+B_{0}(y) \bar{u}+I_{0}\left(y, v_{0}\right)\right], \quad t \in\left[0, \frac{T}{\varepsilon}\right), \\
y\left(0, \bar{u}(0), \bar{v}_{i}(0)\right)=x_{0},
\end{gathered}
$$

where $\bar{u}$ is the allowable control of the averaging problem (4) that satisfies the same conditions as the allowable control of the exact problem (1), and $v_{0}$ for each $v_{i}$ is selected from the condition a5).

The set of admissible controls $\left(u(t), v_{0}\right)$ of the problem (4) is denoted by $\bar{\Omega}$. The quality criterion of the problem of averaging is as follows:

$$
\bar{J}_{\varepsilon}(\bar{u}, \bar{v})=\varepsilon \int_{0}^{\frac{T}{\varepsilon}}\left[C_{0}(y(t))+F(t, \bar{u})+\psi_{0}\left(y(t), v_{0}\right)\right] d t .
$$

Let us denote

$$
J_{\mathcal{\varepsilon}}^{*}=\inf _{\left(u(t), v_{i}\right) \in \Omega} J_{\varepsilon}(u, v), \quad \bar{J}_{\varepsilon}^{*}=\inf _{\left(u(t), v_{0}\right) \in \Omega} \bar{J}_{\mathcal{\varepsilon}}(\bar{u}, \bar{v}) .
$$

The purpose of this work is to prove for the problem of optimal control the following statement: for an arbitrary $\eta>0$ there exists $\varepsilon_{0}=\varepsilon_{0}(\eta)$ such that for $\varepsilon<\varepsilon_{0}$ the inequality

$$
\left|J_{\mathcal{\varepsilon}}^{*}-J_{\mathcal{\varepsilon}}\left(\bar{u}^{*}, v_{0}^{*}\right)\right| \leq \eta
$$

holds; $\bar{u}^{*}, v_{0}^{*}$ is the optimal control pair for the problem of averaging, i.e., the optimal control of the problem of averaging is almost optimal for the exact one.

For the averaged system (4) we assume that the following condition fulfilled.

(A) If the control $\bar{u}$ satisfies the estimate

$$
\varepsilon \int_{0}^{\frac{T}{\varepsilon}}|\bar{u}(t)| d t \leq R
$$

where $R>0$ does not depend on $\varepsilon, \bar{u}$, then there is $\varepsilon_{0}=\varepsilon_{0}(R)$ such that for $0<\varepsilon<\varepsilon_{0}$ the solution $y\left(t, \bar{u}, v_{0}\right)$ of the averaged Cauchy problem for $t \in\left[0, \frac{T}{\varepsilon}\right]$ belongs to the region $D$ together with some $\rho$-neighborhood, and $\rho$ does not depend on $\varepsilon, \bar{u}, v_{0}$.

The following theorem holds.

Theorem. Under conditions 1.1)-1.7) and condition (A) there exists $\varepsilon_{0}>0$ such that for $0<\varepsilon<\varepsilon_{0}$ the exact and averaged control problems have solutions, and for an arbitrary $\eta>0$ there exists $\varepsilon_{1}=\varepsilon_{1}(\eta) \leq \varepsilon_{0}$ such that the inequality

$$
\left|J_{\mathcal{\varepsilon}}^{*}-J_{\mathcal{E}}\left(\bar{u}^{*}, v_{0}^{*}\right)\right| \leq \eta,
$$

holds with $0<\varepsilon<\varepsilon_{1}$, where $\left(\bar{u}^{*}, v_{0}^{*}\right)$ is the optimal control of the averaging system.

\section{THE AVERAGING LEMMA}

In this section, we will prove the lemma on the proximity of the solutions of the initial and averaged systems, which is a generalization of Bogolyubov's first theorem for impulse systems [4], in the case of the dependence of the right-hand sides on functional parameters. This lemma plays an essential role in proving the main result of the work. 
Lemma. If the conditions 1.1)-1.7) and condition (A) are met for an arbitrary $\eta>0$, there exists $\varepsilon_{0}=\varepsilon_{0}(R, \eta)$ such that for $0<\varepsilon<\varepsilon_{0}$ the solution $x(t, u, v)$ of the Cauchy problem of the exact system (1) was determined on $\left[0, \frac{T}{\varepsilon}\right]$ and the following estimate holds

$$
\left|x(t, u, v)-y\left(\varepsilon t, u, v_{0}\right)\right| \leq \eta, \quad t \in\left[0, \frac{T}{\varepsilon}\right]
$$

for each admissible control $(u, v)$ of the exact problem and for each admissible control $\left(u, v_{0}\right)$ of the averaged problem, where $u$ satisfies condition (A), and $v_{0}$ is selected from condition a5).

Proof. We take an arbitrary $0<\eta<\frac{\rho}{2}$ and fix it. Going in (1) and (4) to integral images, we have for an arbitrary $t \geq 0$ to the moment of output of the solution $x(t)=x(t, u, v)$ on the boundary of the domain $D$ :

$$
x(t)=x_{0}+\varepsilon \int_{0}^{t}[A(s, x(s))+B(s, x(s)) u(s)] d s+\varepsilon \sum_{0 \leq t_{i}<t} I_{i}\left(x\left(t_{i}\right), v_{i}\right),
$$

and for $y(t)=y\left(t, u, v_{0}\right)$ :

$$
y(t)=x_{0}+\varepsilon \int_{0}^{t}\left[A_{0}(y(s))+B_{0}(y(s)) u(s)+I_{0}\left(y(s), v_{0}\right)\right] d s .
$$

Then

$$
\begin{aligned}
x(t)-y(t) & =\varepsilon \int_{0}^{t}[A(s, x(s))-A(s, y(s))] d s+\varepsilon \int_{0}^{t}\left[A(s, y(s))-A_{0}(y(s))\right] d s \\
& +\varepsilon \int_{0}^{t}[B(s, x(s))-B(s, y(s))] u(s) d s+\varepsilon \int_{0}^{t}\left[B(s, y(s))-B_{0}(y(s))\right] u(s) d s \\
& +\varepsilon\left(\sum_{0 \leq t_{i}<t} I_{i}\left(x\left(t_{i}\right), v_{i}\right)-\sum_{0 \leq t_{i}<t} I_{i}\left(y\left(t_{i}\right), v_{i}\right)\right)+\varepsilon\left(\sum_{0 \leq t_{i}<t} I_{i}\left(y\left(t_{i}\right), v_{i}\right)-\sum_{0 \leq t_{i}<t} I_{i}\left(y\left(t_{i}\right), v_{0}\right)\right) \\
& +\varepsilon\left(\sum_{0 \leq t_{i}<t} I_{i}\left(y\left(t_{i}\right), v_{0}\right)-\int_{0}^{t} I_{0}\left(y(s), v_{0}\right) d s\right),
\end{aligned}
$$

whence, to the first, third and fifth terms, applying Lipschitz's conditions 1.3) and 1.6), to the fourth terms, applying the Hölder's inequality for integrals, we obtain

$$
\begin{aligned}
|x(t)-y(t)| & \leq \varepsilon L \int_{0}^{t}(1+|u(s)|)|x(s)-y(s)| d s+\varepsilon\left|\int_{0}^{t}\left[A(s, y(s))-A_{0}(y(s))\right] d s\right| \\
& +\left(\varepsilon \int_{0}^{t}\left|B(s, y(s))-B_{0}(y(s))\right|^{q} d s\right)^{\frac{1}{q}}\left(\varepsilon \int_{0}^{t}|u(s)|^{p} d s\right)^{\frac{1}{p}} \\
& +\varepsilon L \sum_{0 \leq t_{i}<t}\left|x\left(t_{i}\right)-y\left(t_{i}\right)\right|+\varepsilon\left(\sum_{0 \leq t_{i}<t} I_{i}\left(y\left(t_{i}\right), v_{i}\right)-\sum_{0 \leq t_{i}<t} I_{i}\left(y\left(t_{i}\right), v_{0}\right)\right) \\
& +\varepsilon\left|\sum_{0 \leq t_{i}<t} I_{i}\left(y\left(t_{i}\right), v_{0}\right)-\int_{0}^{t} I_{0}\left(y(s), v_{0}\right) d s\right| .
\end{aligned}
$$

That is,

$$
\begin{aligned}
|x(t)-y(t)| & \leq \varepsilon L \int_{0}^{t}(1+|u(s)|)|x(s)-y(s)| d s+\varepsilon\left|\int_{0}^{t}\left[A(s, y(s))-A_{0}(y(s))\right] d s\right| \\
& +C^{\frac{1}{p}}\left(\varepsilon \int_{0}^{t} \| B(s, y(s))-B_{0}(y(s))||^{q} d s\right)^{\frac{1}{q}}+\varepsilon L \sum_{0 \leq t_{i}<t}\left|x\left(t_{i}\right)-y\left(t_{i}\right)\right| \\
& +\varepsilon\left(\sum_{0 \leq t_{i}<t} I_{i}\left(y\left(t_{i}\right), v_{i}\right)-\sum_{0 \leq t_{i}<t} I_{i}\left(y\left(t_{i}\right), v_{0}\right)\right)+\varepsilon\left|\sum_{0 \leq t_{i}<t} I_{i}\left(y\left(t_{i}\right), v_{0}\right)-\int_{0}^{t} I_{0}\left(y(s), v_{0}\right) d s\right| .
\end{aligned}
$$


According to condition a5), we choose and fix such $T_{0}$ and $N_{0}=N_{0}\left(T_{0}\right)$ that at $t_{i}>T_{0}$, $i \geq N_{0}$, the inequality $\left|v_{i}-v_{0}\right|<\frac{\eta}{4}$ is fulfilled.

Let us evaluate the term

$$
I=\varepsilon\left(\sum_{0 \leq t_{i}<t} I_{i}\left(y\left(t_{i}\right), v_{i}\right)-\sum_{0 \leq t_{i}<t} I_{i}\left(y\left(t_{i}\right), v_{0}\right)\right) .
$$

Given conditions 1.5), 1.6), as well as condition a5), we have

$$
\begin{aligned}
I & \leq \varepsilon \sum_{0 \leq t_{i}<T_{0}}\left|I_{i}\left(y\left(t_{i}\right), v_{i}\right)-I_{i}\left(y\left(t_{i}\right), v_{0}\right)\right|+\varepsilon \sum_{T_{0} \leq t_{i}<t}\left|I_{i}\left(y\left(t_{i}\right), v_{i}\right)-I_{i}\left(y\left(t_{i}\right), v_{0}\right)\right| \\
& \leq \varepsilon 2 M \widetilde{C} T_{0}+\varepsilon \frac{T}{\varepsilon} \widetilde{C} \frac{\eta}{4}<\frac{\eta}{3} .
\end{aligned}
$$

So

$$
\begin{aligned}
|x(t)-y(t)| & \leq \varepsilon L \int_{0}^{t}(1+|u(s)|)|x(s)-y(s)| d s+\varepsilon\left|\int_{0}^{t}\left[A(s, y(s))-A_{0}(y(s))\right] d s\right| \\
& +C^{\frac{1}{p}}\left(\varepsilon \int_{0}^{t}|| B(s, y(s))-B_{0}(y(s))||^{q} d s\right)^{\frac{1}{q}}+\varepsilon L \sum_{0 \leq t_{i}<t}\left|x\left(t_{i}\right)-y\left(t_{i}\right)\right|+\frac{\eta}{3} \\
& +\varepsilon\left|\sum_{0 \leq t_{i}<t} I_{i}\left(y\left(t_{i}\right), v_{0}\right)-\int_{0}^{t} I_{0}\left(y(s), v_{0}\right) d s\right| .
\end{aligned}
$$

Let us evaluate the last two integrals separately:

$$
I_{1}=\varepsilon\left|\int_{0}^{t}\left[A(s, y(s))-A_{0}(y(s))\right] d s\right|
$$

and

$$
I_{2}=\varepsilon \int_{0}^{t}\left\|B(s, y(s))-B_{0}(y(s))\right\|^{q} d s
$$

We divide $\left[0, \frac{T}{\varepsilon}\right]$ into $n$ equal parts by points $\tau_{i}$. For an arbitrary $t \in\left[\tau_{i}, \tau_{i+1}\right]$ :

$$
\begin{aligned}
I_{1} & \leq \varepsilon\left|\int_{0}^{t}\left[A(s, y(s))-A_{0}(y(s))\right] d s\right| \leq \varepsilon\left|\sum_{i=0}^{n-1} \int_{\tau_{i}}^{\tau_{i+1}}\left[A(s, y(s))-A_{0}(y(s))\right] d s\right| \\
& \leq \varepsilon \sum_{i=0}^{n-1} \int_{\tau_{i}}^{\tau_{i+1}}\left(\left|A(s, y(s))-A\left(s, y\left(\tau_{i}\right)\right)\right|+\left|A_{0}\left(y\left(\tau_{i}\right)\right)-A_{0}(y(s))\right|\right) d s \\
& \leq 2 L \varepsilon \sum_{i=0}^{n-1} \int_{\tau_{i}}^{\tau_{i+1}}\left|y(s)-y\left(\tau_{i}\right)\right| d s+\varepsilon\left|\sum_{i=0}^{n-1} \int_{\tau_{i}}^{\tau_{i+1}}\left(A\left(s, y\left(\tau_{i}\right)\right)-A_{0}\left(y\left(\tau_{i}\right)\right)\right) d s\right| .
\end{aligned}
$$

For the difference $y(s)-y\left(\tau_{i}\right)$ we obtain:

$$
\begin{aligned}
\left|y(s)-y\left(\tau_{i}\right)\right| & =\varepsilon\left|\int_{\tau_{i}}^{s}\left[A_{0}(y(\tau))+B_{0}(y(\tau)) u(\tau)\right] d \tau\right| \\
& \leq \varepsilon \int_{\tau_{i}}^{s}\left(\left|A_{0}(y(\tau))\right|+\left|B_{0}(y(\tau))\right| \cdot|| u(\tau)||\right) d \tau \\
& \leq \varepsilon M \int_{\tau_{i}}^{\tau_{i+1}}(1+|u(\tau)|) d \tau \leq \frac{M T}{n}+M \varepsilon\left(\varepsilon \int_{\tau_{i}}^{\tau_{i+1}}|u(s)|^{p} d s\right)^{\frac{1}{p}}\left(\frac{T}{\varepsilon n}\right)^{\frac{1}{q}} \\
& \leq \frac{M T}{n}+M\left(\frac{T}{n}\right)^{\frac{1}{q}}\left(\varepsilon \int_{\tau_{i}}^{\tau_{i+1}}|u(s)|^{p} d s\right)^{\frac{1}{p}} .
\end{aligned}
$$


Using the condition (A), we have the following estimate

$$
\left|y(s)-y\left(\tau_{i}\right)\right| \leq \frac{M T}{n}+M\left(\frac{T}{n}\right)^{\frac{1}{q}} R^{\frac{1}{p}}
$$

So

$$
\begin{aligned}
I_{11}=2 L \varepsilon \sum_{i=0}^{n-1} \int_{\tau_{i}}^{\tau_{i+1}}\left|y(s)-y\left(\tau_{i}\right)\right| d s & \leq 2 L \varepsilon \sum_{i=0}^{n-1}\left[\frac{M T}{n}+M\left(\frac{T}{n}\right)^{\frac{1}{q}} R^{\frac{1}{p}}\right]\left(\frac{T}{n \varepsilon}\right) \\
& \leq 2 L M T\left(\frac{T}{n}+\left(\frac{T}{n}\right)^{\frac{1}{q}} R^{\frac{1}{p}}\right) .
\end{aligned}
$$

Let us fix $n$ so that the inequality

$$
2 \operatorname{LMT}\left(\frac{T}{n}+\left(\frac{T}{n}\right)^{\frac{1}{q}} R^{\frac{1}{p}}\right) \leq \frac{\eta}{4}
$$

holds.

Due to conditions 1.1) and 1.8) it is possible to construct such a monotonically decreasing function $\varphi(t) \rightarrow 0, t \rightarrow \infty$, that the following inequalities hold uniformly over $y \in D, v \in V$ :

$$
\begin{aligned}
\left|\int_{0}^{t}\left[A(s, y)-A_{0}(y)\right] d s\right| & \leq \frac{t \varphi(t)}{2}, \\
\left|\sum_{0<t_{i}<T} I_{i}(x, v)-I_{0}(x, v) T\right| & \leq \frac{\varphi(T)}{2} T .
\end{aligned}
$$

If $t \in\left[\tau_{i}, \tau_{i+1}\right]$ (except $t \in\left[0, \tau_{1}\right], \tau_{1}<\tau_{2}$ ), then we have

$$
\begin{aligned}
I_{12} & \leq \varepsilon \sum_{i=0}^{n-1}\left(\left|\int_{0}^{\tau_{i+1}}\left(A\left(s, y\left(\tau_{i}\right)\right)-A_{0}\left(y\left(\tau_{i}\right)\right)\right) d s\right|+\left|\int_{0}^{\tau_{i}}\left(A\left(s, y\left(\tau_{i}\right)\right)-A_{0}\left(y\left(\tau_{i}\right)\right)\right) d s\right|\right) \\
& \leq \varepsilon \sum_{i=0}^{n-1}\left(\tau_{i+1} \varphi\left(\tau_{i+1}\right)+\tau_{i} \varphi\left(\tau_{i}\right)\right) \leq \varepsilon \sum_{i=0}^{n-1} \frac{T}{\varepsilon} \varphi\left(\frac{T}{n \varepsilon}\right) \leq 2 n T \varphi\left(\frac{T}{n \varepsilon}\right) .
\end{aligned}
$$

Since $n$ is fixed, then for $\varepsilon<\varepsilon_{0}$, we obtain:

$$
\varepsilon\left|\int_{0}^{t}\left(A\left(s, y\left(\tau_{i}\right)\right)-A_{0}\left(y\left(\tau_{i}\right)\right)\right) d s\right| \leq \frac{\eta}{8}
$$

If $t \in\left[0, \tau_{1}\right]$, then

$$
\varepsilon\left|\int_{0}^{t}\left[A\left(s, y\left(\tau_{i}\right)\right)-A_{0}\left(y\left(\tau_{i}\right)\right)\right] d s\right| \leq \varepsilon \int_{0}^{t}\left(\left|A\left(s, y\left(\tau_{i}\right)\right)\right|+\left|A_{0}\left(y\left(\tau_{i}\right)\right)\right|\right) d s \leq 2 M \frac{T}{\varepsilon n}=\frac{2 M T}{n} .
$$

In this case, we choose $n$ so that in addition to (8) the inequality

$$
\frac{2 M T}{n} \leq \frac{\eta}{8}
$$

is also will be satisfied. 
When $n$ is selected, the function $\varphi\left(\frac{T}{n \varepsilon}\right) \rightarrow 0$ for $\varepsilon \rightarrow 0$, and therefore, for an arbitrary $\eta>0$ there exists $\varepsilon_{0}>0$ such that for $0<\varepsilon<\varepsilon_{0}$ we have:

$$
\varepsilon\left|\sum_{i=0}^{n-1} \int_{\tau_{i}}^{\tau_{i+1}}\left(A\left(s, y\left(\tau_{i}\right)\right)-A_{0}\left(y\left(\tau_{i}\right)\right)\right) d s\right| \leq \frac{\eta}{4}
$$

So, $I_{1}=\left|\int_{0}^{t}\left(A(s, y(s))-A_{0}(y(s))\right) d s\right| \leq \frac{\eta}{2}$.

Now, let us evaluate the expression $I_{2}$.

Again, we divide the segment $\left[0, \frac{T}{\varepsilon}\right]$ on $n$ equal parts by the points $\tau_{i}$. For an arbitrary $t \in\left[\tau_{i}, \tau_{i+1}\right]$, we have:

$$
\begin{aligned}
I_{2} & =\sum_{i=0}^{n-1} \varepsilon \int_{\tau_{i}}^{\tau_{i+1}}\left\|B(s, y(s))-B_{0}(y(s))\right\|^{q} d s \\
& =\sum_{i=0}^{n-1} \varepsilon \int_{\tau_{i}}^{\tau_{i+1}}\left\|B(s, y(s))-B\left(s, y\left(\tau_{i}\right)\right)+B\left(s, y\left(\tau_{i}\right)\right)-B_{0}\left(y\left(\tau_{i}\right)\right)+B_{0}\left(y\left(\tau_{i}\right)-B_{0}(y(s))\right)\right\|^{q} d s .
\end{aligned}
$$

Using Minkowski's inequality, we have:

$$
\begin{aligned}
I_{2} & \leq \varepsilon \sum_{i=0}^{n-1}\left[\left(\int_{\tau_{i}}^{\tau_{i+1}}\left\|B\left(s, y\left(\tau_{i}\right)\right)-B_{0}\left(y\left(\tau_{i}\right)\right)\right\|^{q} d s\right)^{\frac{1}{q}}\right. \\
& \left.+\left(\int_{\tau_{i}}^{\tau_{i+1}}\left\|B(s, y(s))-B\left(s, y\left(\tau_{i}\right)\right)\right\|+\| B_{0}\left(y\left(\tau_{i}\right)-B_{0}(y(s)) \|\right)^{q} d s\right)^{\frac{1}{q}}\right] . \\
I_{2} & \leq \varepsilon \sum_{i=0}^{n-1}\left[\left(\int_{\tau_{i}}^{\tau_{i+1}}\left\|B\left(s, y\left(\tau_{i}\right)\right)-B_{0}\left(y\left(\tau_{i}\right)\right)\right\|^{q} d s\right)^{\frac{1}{q}}\right. \\
& \left.+\left(\int_{\tau_{i}}^{\tau_{i+1}}\left\|B(s, y(s))-B\left(s, y\left(\tau_{i}\right)\right)\right\|+\| B_{0}\left(y\left(\tau_{i}\right)-B_{0}(y(s)) \|\right)^{q} d s\right)^{\frac{1}{q}}\right] .
\end{aligned}
$$

Using condition 1.3) and Iensen's inequality, we obtain:

$$
I_{2} \leq 2^{q-1} \varepsilon \sum_{i=0}^{n-1}\left(2 L^{q} \int_{\tau_{i}}^{\tau_{i+1}}\left|y(s)-y\left(\tau_{i}\right)\right|^{q} d s+\int_{\tau_{i}}^{\tau_{i+1}} \| B\left(s, y\left(\tau_{i}\right)\right)-B_{0}\left(y\left(\tau_{i}\right)\right) \mid{ }^{q} d s\right)
$$

For the difference $\left|y(s)-y\left(\tau_{i}\right)\right|$ we apply the previously obtained estimate, then

$$
\begin{aligned}
I_{2} & \leq 2^{q-1} \mathcal{\varepsilon} \sum_{i=0}^{n-1}\left(2 L^{q} M^{q}\left(\frac{T}{n}+\left(\frac{T}{n}\right)^{\frac{1}{q}} R^{\frac{1}{p}}\right)^{q} \frac{T}{\varepsilon n}+\int_{\tau_{i}}^{\tau_{i+1}}\left\|B\left(s, y\left(\tau_{i}\right)\right)-B_{0}\left(y\left(\tau_{i}\right)\right)\right\|{ }^{q} d s\right) \\
& \leq L^{q} M^{q}\left(\frac{T}{n}+\left(\frac{T}{n}\right)^{\frac{1}{q}} R^{\frac{1}{p}}\right)^{q} T+2^{q-1} \varepsilon \sum_{i=0}^{n-1} \int_{\tau_{i}}^{\tau_{i+1}}\left\|B\left(s, y\left(\tau_{i}\right)\right)-B_{0}\left(y\left(\tau_{i}\right)\right)\right\|^{q} d s .
\end{aligned}
$$


We fix $n$ so that the condition

$$
\left(2 \operatorname{LM}\left(\frac{T}{n}+\left(\frac{T}{n}\right)^{\frac{1}{q}} R^{\frac{1}{p}}\right)\right)^{q} T \leq \frac{1}{2}\left(\frac{\eta}{2 R}\right)^{q}
$$

is fullfiled. Estimate the term

$$
I_{21}=2^{q-1} \varepsilon \sum_{i=0}^{n-1} \int_{\tau_{i}}^{\tau_{i+1}}\left\|B\left(s, y\left(\tau_{i}\right)\right)-B_{0}\left(y\left(\tau_{i}\right)\right)\right\|^{q} d s .
$$

By condition 1.1) the inequality $\int_{0}^{t}\left\|B(s, y)-B_{0}(y)\right\|^{q} d s \leq t \varphi(t)$ holds uniformly over $y \in D$. If $t \in\left[\tau_{i}, \tau_{i+1}\right]$ (expect $t \in\left[0, \tau_{1}\right], \tau_{1}<\tau_{2}$ ), then we have

$$
\begin{aligned}
I_{21} & \leq 2^{q-1} \varepsilon \sum_{i=0}^{n-1}\left(\int_{\tau_{i}}^{\tau_{i+1}}\left\|B\left(s, y\left(\tau_{i}\right)\right)-B_{0}\left(y\left(\tau_{i}\right)\right)\right\|^{q} d s-\int_{0}^{\tau_{i}}\left\|B\left(s, y\left(\tau_{i}\right)\right)-B_{0}\left(y\left(\tau_{i}\right)\right)\right\|^{q} d s\right) \\
& \leq 2^{q-1} \varepsilon \sum_{i=0}^{n-1}\left(\tau_{i+1} \varphi\left(\tau_{i+1}\right)+\tau_{i} \varphi\left(\tau_{i}\right)\right) \leq 2^{q-1} \varepsilon \sum_{i=0}^{n-1} \frac{T}{\varepsilon} \varphi\left(\frac{T}{n \varepsilon}\right) \leq 2^{q} n T \varphi\left(\frac{T}{n \varepsilon}\right),
\end{aligned}
$$

since $n$ is fixed, then $\varphi\left(\frac{T}{n \varepsilon}\right) \rightarrow 0$ as $\varepsilon \rightarrow 0$.

If $t \in\left[0, \tau_{1}\right]$, then

$$
\varepsilon \int_{0}^{t}\left\|B\left(s, y\left(\tau_{i}\right)\right)-B_{0}(y(\tau))\right\|^{q} d s \leq \varepsilon \int_{0}^{t}\left(\left\|B\left(s, y\left(\tau_{i}\right)\right)\right\|+\left\|B_{0}\left(y\left(\tau_{i}\right)\right)\right\|\right)^{q} d s \leq \frac{2^{q} M^{q} T}{n} .
$$

We fix $n$ so that in addition to (9) the inequality $\frac{2^{q} M^{q} T}{n} \leq \frac{\eta}{8}$ also holds. Thus, by choosing a sufficiently small $\varepsilon$ we have estimate $2^{q} n T \varphi\left(\frac{T}{n \varepsilon}\right)^{n}+\frac{2^{q} M^{q} T}{n} \leq \frac{1}{2}\left(\frac{\eta}{2 R}\right)^{q}$, and hence,

$$
\left(\varepsilon \int_{0}^{t}\left\|B(s, y(s))-B_{0}(y(s))\right\|^{q} d s\right)^{\frac{1}{q}} \leq \frac{\eta}{2 R} .
$$

It remains to estimate the value

$$
\varepsilon\left|\sum_{0 \leq \tau_{i}<t} I_{i}\left(y\left(\tau_{i}\right), v_{0}\right)-\int_{0}^{t} I_{0}\left(y(s), v_{0}\right) d s\right| .
$$

To do this, we again divide the segment $\left[0, \frac{T}{\varepsilon}\right]$ by the points $\left\{\tau_{k}\right\}$ into $n$ equal parts. We have

$$
\begin{aligned}
\varepsilon \mid \sum_{0 \leq t_{i}<t} I_{i}\left(y\left(t_{i}\right), v_{0}\right) & -\int_{0}^{t} I_{0}\left(y(s), v_{0}\right) d s \mid \\
& =\varepsilon\left|\sum_{k=0}^{n-1}\left(\sum_{\tau_{k} \leq t_{i}<\tau_{k+1}} I_{i}\left(y\left(t_{i}\right), v_{0}\right)-\int_{\tau_{k}}^{\tau_{k+1}} I_{0}\left(y(s), v_{0}\right) d s\right)\right| .
\end{aligned}
$$

Let us estimate each of terms in the last sum:

$$
\begin{aligned}
\varepsilon \mid \sum_{\tau_{k} \leq t_{i}<\tau_{k+1}} I_{i}\left(y\left(t_{i}\right), v_{0}\right) & -\int_{\tau_{k}}^{\tau_{k+1}} I_{0}\left(y(s), v_{0}\right) d s \mid \\
\leq \varepsilon\left|\sum_{\tau_{k} \leq t_{i}<\tau_{k+1}} I_{i}\left(y\left(t_{i}\right), v_{0}\right)-I_{0}\left(y\left(\tau_{k}\right), v_{0}\right)\left(\tau_{k+1}-\tau_{k}\right)\right| & \quad+\varepsilon\left|I_{0}\left(y\left(\tau_{k}\right), v_{0}\right)\left(\tau_{k+1}-\tau_{k}\right)-\int_{\tau_{k}}^{\tau_{k+1}} I_{0}\left(y(s), v_{0}\right) d s\right| .
\end{aligned}
$$


We separately evaluate each term from (11):

$$
\begin{aligned}
& \varepsilon\left|I_{0}\left(y\left(\tau_{k}\right), v_{0}\right)\left(\tau_{k+1}-\tau_{k}\right)-\int_{\tau_{k}}^{\tau_{k+1}} I_{0}\left(y(s), v_{0}\right) d s\right| \\
& =\varepsilon\left|\int_{\tau_{k}}^{\tau_{k+1}}\left(I_{0}\left(y\left(\tau_{k}\right), v_{0}\right)-I_{0}\left(y(s), v_{0}\right)\right) d s\right| \leq \varepsilon L \int_{\tau_{k}}^{\tau_{k+1}}\left|y\left(\tau_{k}\right)-y(s)\right| d s .
\end{aligned}
$$

But, by virtue of (7) we have that

$$
\left|y\left(\tau_{k}\right)-y(s)\right| \leq \frac{M T}{n}+M\left(\frac{T}{n}\right)^{\frac{1}{q}} R^{\frac{1}{p}}
$$

Substituting (13) into (12), we have that

$$
\begin{aligned}
\varepsilon \mid I_{0}\left(y\left(\tau_{k}\right), v_{0}\right)\left(\tau_{k+1}-\tau_{k}\right) & -\int_{\tau_{k}}^{\tau_{k+1}} I_{0}\left(y(s), v_{0}\right) d s \mid \\
& \leq \varepsilon L \frac{T}{\varepsilon n}\left(\frac{M T}{n}+M\left(\frac{T}{n}\right)^{\frac{1}{q}} R^{\frac{1}{p}}\right)=\frac{T L}{n}\left(\frac{M T}{n}+M\left(\frac{T}{n}\right)^{\frac{1}{q}} R^{\frac{1}{q}}\right) .
\end{aligned}
$$

It remains to evaluate the first term in (11). We have

$$
\begin{aligned}
\varepsilon\left|\sum_{\tau_{k} \leq t_{i}<\tau_{k+1}} I_{i}\left(y\left(t_{i}\right), v_{0}\right)-I_{0}\left(y\left(\tau_{k}\right), v_{0}\right)\left(\tau_{k+1}-\tau_{k}\right)\right| \\
\quad \leq \varepsilon \sum_{\tau_{k} \leq t_{i}<\tau_{k+1}} L\left|y\left(t_{i}\right)-y\left(\tau_{k}\right)\right|+\varepsilon\left|\sum_{\tau_{k} \leq t_{i}<\tau_{k+1}} I_{i}\left(y\left(\tau_{k}\right), v_{0}\right)-I_{0}\left(y\left(\tau_{k}\right), v_{0}\right)\left(\tau_{k+1}-\tau_{k}\right)\right| \\
\quad \leq \frac{T L}{n}\left(\frac{M T}{n}+M\left(\frac{T}{n}\right)^{\frac{1}{q}} R^{\frac{1}{p}}\right)+\varepsilon\left|\sum_{\tau_{k} \leq t_{i}<\tau_{k+1}} I_{i}\left(y\left(\tau_{k}\right), v_{0}\right)-I_{0}\left(y\left(\tau_{k}, v_{0}\right)\right)\left(\tau_{k+1}-\tau_{k}\right)\right|,
\end{aligned}
$$

according to condition (14). Then (10) is evaluated by the following expression:

$$
T L\left(\frac{M T}{n}+N\left(\frac{T}{n}\right)^{\frac{1}{q}} R^{\frac{1}{p}}\right)+\varepsilon\left|\sum_{i=0}^{n-1}\left(\sum_{\tau_{k} \leq t_{i}<\tau_{k+1}} I_{i}\left(y\left(\tau_{k}\right), v_{0}\right)\left(\tau_{k+1}-\tau_{k}\right)\right)\right| .
$$

Let us evaluate the second term. We have

$$
\begin{aligned}
\varepsilon & \left|\sum_{k=0}^{n-1}\left(\sum_{\tau_{k} \leq t_{i}<\tau_{k+1}} I_{i}\left(y\left(\tau_{k}\right), v_{0}\right)-I_{0}\left(y\left(\tau_{k}\right), v_{0}\right)\left(\tau_{k+1}-\tau_{k}\right)\right)\right| \\
& =\varepsilon\left|\sum_{k=0}^{n-1}\left(\sum_{0 \leq t_{i}<\tau_{k+1}} I_{i}\left(y\left(\tau_{k}\right), v_{0}\right)-I_{0}\left(y\left(\tau_{k}\right), v_{0}\right) \tau_{k+1}-\sum_{0<t_{i}<\tau_{k}} I_{i}\left(y\left(\tau_{k}\right), v_{0}\right)-I_{0}\left(y\left(\tau_{k}\right), v_{0}\right) \tau_{k}\right)\right| \\
& \left.\leq \varepsilon \sum_{k=0}^{n-1} \mid \sum_{0 \leq t_{i}<\tau_{k+1}} I_{i} y\left(\tau_{k}\right), v_{0}\right)-I_{0}\left(y\left(\tau_{k}\right), v_{0}\right) \tau_{k+1}\left|+\varepsilon \sum_{k=0}^{n-1}\right| \sum_{0<t_{i}<\tau_{k}} I_{i}\left(y\left(\tau_{k}\right), v_{0}\right)-I_{0}\left(y\left(\tau_{k}\right), v_{0}\right) \tau_{k} \mid \\
& \leq \varepsilon \sum_{k=0}^{n-1} \frac{\tau_{k+1} \varphi\left(\tau_{k+1}\right)}{2}+\varepsilon \sum_{k=0}^{n-1} \frac{\tau_{k} \varphi\left(\tau_{k}\right)}{2} \leq \varepsilon \sum_{k=0}^{n-1} \frac{T}{\varepsilon} \varphi\left(\frac{T}{\varepsilon n}\right)=n T \varphi\left(\frac{T}{\varepsilon n}\right) .
\end{aligned}
$$


We choose $\varepsilon$ so small that for the selected fixed $n$ the following inequality holds:

$$
n T \varphi\left(\frac{T}{\varepsilon n}\right) \leq \frac{\eta}{4} e^{-L T} e^{-M L T}
$$

and we fix such $n$.

Returning now to (6), given the choice of $\varepsilon$, we have

$$
|x(t)-y(t)| \leq \eta+\varepsilon L \int_{0}^{t}(1+|u(s)|)|x(s)-y(s)| d s+\varepsilon L \sum_{0 \leq t_{i}<t}\left|x\left(t_{i}\right)-y\left(t_{i}\right)\right|,
$$

or

$$
|x(t)-y(t)| \leq \eta+\int_{0}^{t} \varepsilon L(1+|u(s)|)|x(s)-y(s)| d s+\sum_{0 \leq t_{i}<t} \varepsilon L\left|x\left(t_{i}\right)-y\left(t_{i}\right)\right| .
$$

From the analogue of the Gronwall-Bellman lemma [5, p. 12] we obtain

$$
|x(t)-y(t)| \leq \eta \prod_{0<\tau_{i}<t}(1+\varepsilon L) e^{\varepsilon L \int_{0}^{t}(1+|u(s)|) d s} .
$$

Take into account that

$$
\begin{aligned}
\varepsilon L \int_{0}^{t}(1+|u(s)|) d s & \leq L T+L \varepsilon \int_{0}^{t}|u(s)| d s \\
& \leq L T+L\left(\varepsilon \int_{0}^{t}|u(s)|^{p} d s\right)^{\frac{1}{p}} T^{\frac{1}{q}} \leq L\left(T+R^{\frac{1}{p}} T^{\frac{1}{q}}\right)
\end{aligned}
$$

and

$$
\prod_{0<\tau_{i}<t}(1+\varepsilon L)=(1+\varepsilon L)^{i(t)} .
$$

But, from condition (2) we have that

$$
(1+\varepsilon L)^{i(t)} \leq(1+\varepsilon L)^{\widetilde{C} \frac{T}{\varepsilon}} \leq e^{\widetilde{C} T L} .
$$

Therefore, $|x(t)-y(t)| \leq \eta e^{L\left(T+R^{\frac{1}{p}} T^{\frac{1}{\eta}}\right)+C T L}$ before leaving the region $D$. However, $y(t)$ lies in the region $D$ together with its $\rho$-neighborhood at $t \in\left[0, \frac{T}{\varepsilon}\right]$, so by choosing a sufficiently small $\eta$ we obtain that $x(t)$ lies in the region $D$ together with $\frac{\rho}{2}$-neighborhood for $t \in\left[0, \frac{T}{\varepsilon}\right]$, therefore, the last inequality valid for all $t \in\left[0, \frac{T}{\varepsilon}\right]$.

The lemma is proved.

\section{PROOF OF THE THEOREM}

First, we prove the existence of a solution of the problem (1) for each fixed $\varepsilon>0$. It is obvious that the sets $\Omega$ and $\bar{\Omega}$ of admissible controls of exact and averaged problems are not empty. Indeed, the control $u(t) \equiv 0$ satisfies condition (A), and then the solution $y\left(t, 0, v_{0}\right)$ of the averaged problem lies in $D$ together with the $\rho$-neighborhood. Therefore, it follows from the averaging lemma that the solution of the exact problem $x\left(t, 0, v_{0}\right)$ also lies in the domain $D$ for sufficiently small $\varepsilon$ and $t \in\left[0, \frac{T}{\varepsilon}\right]$. 
Since due to the conditions the functional $J_{\mathcal{E}}(u, v) \geq 0$, there is a lower bound by all admissible controls. Denote it by $m$. Let $\left\{u^{(n)}(t), v^{(n)}\right\}$ be a corresponding minimizing sequence such that $J\left(u^{(n)}, v^{(n)}\right) \rightarrow m, n \rightarrow \infty$. From conditions (3) we have that

$$
\varepsilon \int_{0}^{\frac{T}{\varepsilon}} a\left|u^{(n)}(t)\right|^{p} d t+\varepsilon \sum_{0<t_{i}<\frac{T}{\varepsilon}} a\left|v_{i}^{(n)}\right|^{p} \leq m+1
$$

The latter means a week compactness of the sequence $u^{(n)}(t)$ in $L_{p}\left(0, \frac{T}{\varepsilon}\right)$.

From condition (2) it follows that $i\left(\frac{T}{\varepsilon}\right) \leq \widetilde{C} \cdot \frac{T}{\varepsilon}$, and hence the number of impulses for each fixed $\varepsilon>0$ is limited, let it be equal to $N_{1}$.

Therefore, the number of vectors $v^{(n)}$ in (15) is finite. From here and from the weak compactness of the subsequence $u^{(n)}(t)$ it implies the existence of a weakly convergent in $L_{p}\left(0, \frac{T}{\varepsilon}\right)$ subsequence $u^{\left(n_{k}\right)}(t)$ and convergent in $R^{i\left(\frac{T}{\varepsilon}\right)}$ subsequence $v_{i}^{\left(n_{m}\right)}(t)$. It is clear that they can be chosen so that the numbers $n_{k}$ and $n_{m}$ match. Then, without losing generality, we can assume that $u^{(n)}(t)$ and $v_{i}^{(n)}$ are convergent. Therefore, $u^{(n)}(t) \stackrel{w}{\longrightarrow} u^{*}(t) \subset L_{p}\left(0, \frac{T}{\varepsilon}\right)$, and $v_{i}^{(n)} \rightarrow v_{i}^{*}$, $n \rightarrow \infty$ strongly in $R^{r}, i=\overline{1, i\left(\frac{T}{\varepsilon}\right)}$.

From the closedness of $V$ it follows, that $v_{i}^{*} \in V, i=1,2, \ldots, N$.

According to Mazur's lemma [10, p. 173] there is a convex combination

$$
b_{k}(t)=\sum_{i=1}^{n(k)} \alpha_{i(k)} u^{(i)}(t)
$$

of elements $u^{(i)}(t) \in U$, with $\alpha_{i} \geq 0, \sum_{i=1}^{n(k)} \alpha_{i}=1$, such that in $L_{p}$ we have the convergence $b_{k} \rightarrow u^{*}, k \rightarrow \infty$. Thus, there exists a subsequence $b_{k_{l}}$ convergent on $\left[0, \frac{T}{\varepsilon}\right]$ almost everywhere with respect to Lebesgue measure, such that $b_{k_{l}}(t) \rightarrow u^{*}(t), l \rightarrow \infty$ for almost all $t$. Since $U$ is a convex and closed set, then $\sum_{i=1}^{n(k)} \alpha_{i} u^{(i)}(t) \in U$, and $u^{*}(t) \in U$ are for almost all $t$. Then it follows from the closedness of the set $U$ that $u^{*} \in U$ for almost all $t \in\left[0, \frac{T}{\varepsilon}\right]$.

We now estimate the solutions $x^{(n)}(t)$, which correspond to the controls $\left(u^{(n)}(t), v_{i}^{(n)}\right)$. For $q=\frac{p}{p-1}$ we have

$$
\begin{aligned}
\left|x^{(n)}(t)\right|^{q} & \leq\left(\left|x_{0}\right|+\varepsilon \int_{0}^{t}\left|A\left(s, x^{(n)}(s)\right)+B\left(s, x^{(n)}(s)\right)\right| \cdot\left|u^{(n)}(s)\right| d s+\varepsilon \sum_{0 \leq t_{i}<t} I_{0 i}\left(x_{i}^{(n)}\left(t_{i}\right), v_{i}^{(n)}\right)\right)^{q} \\
& \leq 3^{q}\left(\left|x_{0}\right|^{q}+\left(\varepsilon \int_{0}^{t}\left\{K\left(1+\left|x^{(n)}(s)\right|\right)+K\left(1+\left|x^{(n)}(s)\right|\right) \cdot\left|u^{(n)}(s)\right|\right\} d s\right)^{q}\right. \\
& \left.+\varepsilon\left(\sum_{0 \leq t_{i}<t} K\left(1+\left|x_{i}^{(n)}\left(t_{i}\right)\right|\right)\right)^{q}\right) .
\end{aligned}
$$


Let us evaluate each of the terms separately.

$$
\begin{aligned}
\left(\varepsilon \int _ { 0 } ^ { t } \left\{K\left(1+\left|x^{(n)}(s)\right|\right)\right.\right. & \left.\left.+K\left(1+\left|x^{(n)}(s)\right|\right) \cdot\left|u^{(n)}(s)\right|\right\} d s\right)^{q} \\
& \leq \varepsilon^{q} K^{q} \int_{0}^{t}\left(1+\left|x^{(n)}(s)\right|\right)^{q} d s\left(\int_{0}^{t}\left(1+\left|u^{(n)}(s)\right|\right)^{p} d s\right)^{\frac{q}{p}} \\
& \leq(2 \varepsilon K)^{q}\left(\frac{T}{\varepsilon}+\int_{0}^{t}\left|x^{(n)}(s)\right|^{q} d s\right)\left(\frac{T}{\varepsilon}+\int_{0}^{t}\left|u^{(n)}(s)\right|^{p} d s\right)^{\frac{q}{p}} \\
& \leq\left((2 \varepsilon K)^{q} \frac{T}{\varepsilon}+(2 \varepsilon K)^{q} \int_{0}^{t}\left|x^{(n)}(s)\right|^{q} d s\right)\left(\frac{T}{\varepsilon}+\int_{0}^{t}\left|u^{(n)}(s)\right|^{p} d s\right)^{\frac{q}{p}} .
\end{aligned}
$$

Let $(2 \varepsilon K)^{q} \frac{T}{\varepsilon}=C_{1}$. Since

$$
\left(\frac{T}{\varepsilon}+\int_{0}^{t}\left|u^{(n)}(s)\right|^{p} d s\right)^{\frac{q}{p}} \leq\left(\frac{T}{\varepsilon}+\frac{m}{a}\right)^{\frac{q}{p}}=C_{2},
$$

then continuing inequality (17), we have that the second term in it is estimated by the value

$$
C_{1}+C_{2}(2 \varepsilon K)^{q} \int_{0}^{t}\left|x^{(n)}(s)\right|^{q} d s
$$

To estimate to the sum in (15), we have

$$
\begin{aligned}
\varepsilon \sum_{0 \leq t_{i}<t}\left\{K\left(1+\left|x_{i}^{(n)}\left(t_{i}\right)\right|\right)\right\}^{q} \leq \varepsilon K^{q}\left\{\sum_{0 \leq t_{i}<t}\left(1+\left|x_{i}^{(n)}\left(t_{i}\right)\right|\right) \cdot 1\right\}^{q} \\
\leq \varepsilon K^{q} \sum_{0 \leq t_{i}<t}\left(1+\left|x_{i}^{(n)}\left(t_{i}\right)\right|\right)^{q} \cdot\left(\sum_{0 \leq t_{i}<t}|1|^{p}\right)^{\frac{q}{p}} \\
\leq \varepsilon \widetilde{C}^{\frac{q}{p}} \cdot\left(\frac{T}{\varepsilon}\right)^{\frac{q}{p}} \sum_{0 \leq t_{i}<t} K^{q}\left(1+\left|x_{i}^{(n)}\left(t_{i}\right)\right|\right)^{q} \\
\leq \varepsilon \widetilde{C}^{\frac{q}{p}} \cdot\left(\frac{T}{\varepsilon}\right)^{\frac{q}{p}} K^{q} \sum_{0 \leq t_{i}<t} 2^{q-1}\left(1+\left|x_{i}^{(n)}\left(t_{i}\right)\right|\right)^{q} \\
=\varepsilon \widetilde{C}^{\frac{q}{p}} \cdot\left(\frac{T}{\varepsilon}\right)^{\frac{q}{p}} K^{q}\left(\sum_{0 \leq t_{i}<t} 2^{q-1}+2^{q-1} \sum_{0 \leq t_{i}<t}\left|x_{i}^{(n)}\left(t_{i}\right)\right|^{q}\right) \\
\leq \varepsilon \widetilde{C}^{\frac{q}{p}} \cdot\left(\frac{T}{\varepsilon}\right)^{\frac{q}{p}} K^{q}\left(2^{q-1} \cdot i\left(\frac{T}{\varepsilon}\right)+2^{q-1} \sum_{0 \leq t_{i}<t}\left|x_{i}^{(n)}\left(t_{i}\right)\right|^{q}\right) \\
=\varepsilon \widetilde{C}^{\frac{q}{p}} \cdot\left(\frac{T}{\varepsilon}\right)^{\frac{q}{p}} \cdot K^{q} \cdot 2^{q-1} i\left(\frac{T}{\varepsilon}\right)+\varepsilon \widetilde{C}^{\frac{q}{p}} \cdot\left(\frac{T}{\varepsilon}\right)^{\frac{q}{p}} \cdot K^{q} \cdot 2^{q-1} \sum_{0 \leq t_{i}<t}\left|x_{i}^{(n)}\left(t_{i}\right)\right|^{q} \\
=C_{4}+C_{5} \cdot \sum_{0 \leq t_{i}<t}\left|x_{i}^{(n)}\left(t_{i}\right)\right|^{q},
\end{aligned}
$$

where $C_{4}=\varepsilon \widetilde{C}^{\frac{q}{p}} \cdot\left(\frac{T}{\varepsilon}\right)^{\frac{q}{p}} \cdot K^{q} \cdot 2^{q-1} \cdot i(t), C_{5}=\varepsilon \widetilde{C}^{\frac{q}{p}} \cdot\left(\frac{T}{\varepsilon}\right)^{\frac{q}{p}} \cdot K^{q} \cdot 2^{q-1}$. 
From (16)-(19) we obtain the inequality:

$$
\begin{aligned}
\left|x^{(n)}(t)\right|^{q} & \leq C_{1}+C_{2}(2 K \varepsilon)^{q} \int_{0}^{t}\left|x^{(n)}(s)\right|^{q} d s+C_{4}+C_{5} \sum_{0 \leq t_{i}<t}\left|x^{(n)}\left(t_{i}\right)\right|^{q} \\
& =C_{6}+C_{7} \int_{0}^{t}\left|x^{(n)}(s)\right|^{q} d s+C_{5} \sum_{0 \leq t_{i}<t}\left|x^{(n)}\left(t_{i}\right)\right|^{q},
\end{aligned}
$$

where $C_{6}=C_{1}+C_{4}, C_{7}=C_{2}(2 K \varepsilon)^{q}$.

From the analogue of the Gronwall-Bellman inequality [5, p. 12] we have

$$
\left|x^{(n)}(t)\right|^{q} \leq C_{6} e^{C_{7} \frac{T}{\varepsilon}} \prod_{0 \leq t_{i}<t}\left(1+C_{5}\right) \leq C_{6} e^{C_{7} \frac{T}{\varepsilon}}\left(1+C_{5}\right)^{i(t)}=C_{8}
$$

for $t \in\left[0, \frac{T}{\varepsilon}\right]$.

On the interval $\left[0, t_{1}\right]$ for any two moments of time $t^{\prime}, t^{\prime \prime}, 0 \leq t^{\prime} \leq t^{\prime \prime} \leq t_{1}$, by virtue of 1.7) and (20) we have the existence of constant $M_{1}>0$ such that

$$
\begin{aligned}
\left|x^{(n)}\left(t^{\prime}\right)-x^{(n)}\left(t^{\prime \prime}\right)\right| & =\int_{t^{\prime}}^{t^{\prime \prime}}\left(\left|A\left(s, x^{(n)}(s)\right)\right|+\left|B\left(s, x^{(n)}(s)\right)\right|\left|u^{(n)}(s)\right|\right) d s \\
& \leq \varepsilon M_{1} \int_{t^{\prime}}^{t^{\prime \prime}}\left(1+\left|u^{(n)}(s)\right|\right) d s \\
& \leq \varepsilon M_{1}\left|t^{\prime \prime}-t^{\prime}\right|+M_{1} \varepsilon^{\frac{1}{q}}\left|t^{\prime \prime}-t^{\prime}\right|^{\frac{1}{q}}\left(\varepsilon \int_{t^{\prime}}^{t^{\prime \prime}}\left|u^{(n)}(s)\right|^{p} d s\right)^{\frac{1}{p}} \\
& \leq \varepsilon M_{1}\left|t^{\prime \prime}-t^{\prime}\right|+\varepsilon^{\frac{1}{q}} M_{1} C^{\frac{1}{p}}\left|t^{\prime \prime}-t^{\prime}\right|^{\frac{1}{q}} .
\end{aligned}
$$

The inequality (21) means an equal-degree continuity of the sequence of functions $\left\{x^{(n)}(t)\right\}$ on $\left[0, t_{1}\right]$. Then from (20), (21) it follows the existence of a uniformly convergent subsequence $\left\{x^{\left(k_{1}\right)}(t)\right\}$ of the sequence of functions $\left\{x^{(n)}(t)\right\}$ on $\left[0, t_{1}\right]$. Let $x_{1}(t)$ be its limit.

Denote $x^{\left(k_{1}\right)}\left(t_{1}+0\right)=x^{\left(k_{1}\right)}\left(t_{1}\right)+I_{1}\left(x^{\left(k_{1}\right)}\left(t_{1}\right), v_{1}^{k_{1}}\right)$. Consider the equation

$$
x^{\left(k_{1}\right)}(t)=x^{\left(k_{1}\right)}\left(t_{1}+0\right)+\int_{t_{1}}^{t}\left[A\left(s, x^{\left(k_{1}\right)}(s)\right)+B\left(s, x^{\left(k_{1}\right)}(s)\right) u^{\left(k_{1}\right)}(s)\right] d s
$$

with $t \in\left[t_{1}, t_{2}\right]$.

Similarly to the previous one, the compactness of the sequence $\left\{x^{\left(k_{1}\right)}(t)\right\}$ on $\left[t_{1}, t_{2}\right]$ in a uniform metric is proved. Therefore, there is a subsequence $\left\{x^{\left(k_{2}\right)}(t)\right\}$ of the sequence $\left\{x^{\left(k_{1}\right)}(t)\right\}$ such that $\left\{x^{\left(k_{2}\right)}(t)\right\}$ converges uniformly to $x_{2}(t)$ at $t \in\left[t_{1}, t_{2}\right]$. For $k_{2} \rightarrow \infty$ we have that $x_{2}\left(t_{1}+0\right)=x_{1}\left(t_{1}\right)+I_{1}\left(x_{1}\left(t_{1}\right), v_{1}^{*}\right)$. Next, on the interval $\left[t_{2}, t_{3}\right]$ we consider the equation

$$
x^{\left(k_{2}\right)}(t)=x^{\left(k_{2}\right)}\left(t_{2}+0\right)+\int_{t_{2}}^{t}\left[A\left(s, x^{\left(k_{2}\right)}(s)\right)+B\left(s, x^{k_{2}}(s)\right) u^{\left(k_{2}\right)}(s)\right] d s
$$

Similarly, there is a subsequence $\left\{x^{\left(k_{3}\right)}(t)\right\}$ of the sequence $\left\{x^{\left(k_{2}\right)}(t)\right\}$, such that $\left\{x^{\left(k_{3}\right)}(t)\right\}$ converges uniformly to $x_{3}(t)$ at $t \in\left[t_{2}, t_{3}\right]$. 
Now, let us show that the function

$$
x^{*}(t)=\left\{\begin{array}{cc}
x_{1}(t), \quad t \in\left[0, t_{1}\right], \\
x_{2}(t), \quad t \in\left[t_{1}, t_{2}\right], \\
\cdots \\
x_{i\left(\frac{T}{\varepsilon}\right),} \quad t \in\left[t_{\left.i\left(\frac{T}{\varepsilon}\right), \frac{T}{\varepsilon}\right]}\right.
\end{array}\right.
$$

is the solution of the original problem (1) for the controls $u^{*}(t)$ and $v_{i}^{*}$, i.e. that $x^{*}(t)$ satisfies the equation

$$
x^{*}(t)=x_{0}+\varepsilon \int_{0}^{t}\left[A\left(s, x^{*}(s)\right)+B\left(s, x^{*}(s)\right) u^{*}(s)\right] d s+\varepsilon \sum_{0 \leq t_{i}<\frac{T}{\varepsilon}} I_{0 i}\left(x_{i}^{*}\left(t_{i}\right), v_{i}^{*}\right) .
$$

On the interval $\left[0, t_{1}\right]$ the statement is obvious. We show that for $t \in\left[t_{1}, t_{2}\right]$ the equation

$$
x_{2}(t)=x_{1}\left(t_{1}\right)+I_{1}\left(x\left(t_{1}\right), v_{1}^{*}\right)+\varepsilon \int_{t_{1}}^{t}\left[A\left(s, x_{2}(s)\right)+B\left(s, x_{2}(s)\right) u^{*}(s)\right] d s
$$

is valid.

Since $\lim _{k_{2} \rightarrow \infty} x^{\left(k_{2}\right)}(t)=x_{2}(t)$ uniformly for $t \in\left[t_{1}, t_{2}\right]$ and

$$
x^{\left(k_{2}\right)}(t)=x^{\left(k_{2}\right)}\left(t_{1}+0\right)+\int_{t_{1}}^{t}\left[A\left(s, x^{\left(k_{2}\right)}(s)\right)+B\left(s, x^{\left(k_{2}\right)}(s)\right) u^{\left(k_{2}\right)}(s)\right] d s,
$$

$\left(\left\{k_{2}\right\}\right.$-subsequence of the sequence $\left.\left\{k_{1}\right\}\right)$, then $x^{\left(k_{2}\right)}\left(t_{1}+0\right) \rightarrow x_{2}\left(t_{1}+0\right)$ and

$$
x^{\left(k_{2}\right)}\left(t_{1}+0\right)=x^{\left(k_{2}\right)}\left(t_{1}\right)+I_{1}\left(x^{\left(k_{2}\right)}\left(t_{1}\right), v_{1}^{\left(k_{2}\right)}\right) \rightarrow x_{1}\left(t_{1}\right)+I_{1}\left(x\left(t_{1}\right), v_{1}^{*}\right) .
$$

We now turn in (23) to the boundary at $k_{2} \rightarrow \infty$

$$
x_{2}(t)=x_{1}\left(t_{1}\right)+I_{1}\left(x\left(t_{1}\right), v_{1}^{*}\right)+\varepsilon \lim _{k_{2} \rightarrow \infty} \int_{t_{1}}^{t}\left[A\left(s, x^{\left(k_{2}\right)}(s)\right)+B\left(s, x^{\left(k_{2}\right)}(s)\right) u^{\left(k_{2}\right)}(s)\right] d s .
$$

But, $\lim _{k_{2} \rightarrow \infty} \int_{t_{1}}^{t} A\left(s, x^{\left(k_{2}\right)}(s)\right) d s=\int_{t_{1}}^{t} A\left(s, x_{2}(s)\right) d s$ by the Lebesgue's theorem on major convergence, estimation (20) and linear growth of a function $A$.

Using the limiting relation $\lim _{k_{2} \rightarrow \infty} \int_{t_{1}}^{t} B\left(s, x_{2}(s)\right)\left[u^{\left(k_{2}\right)}(s)-u^{*}(s)\right] d s=0$, and using the relation $\lim _{k_{2} \rightarrow \infty} B\left(s, x^{\left(k_{2}\right)}(s)\right)=B\left(s, x_{2}(s)\right)$, which is uniform, outside some set $S$ of arbitrary small measure, and the inequality

$$
\int_{S}\left|u^{\left(k_{2}\right)}(s)\right| d s \leq\left(\int_{t_{1}}^{t}\left|u^{\left(k_{2}\right)}(s)\right|^{p} d s\right)^{\frac{1}{p}} \cdot(\mu(s))^{\frac{1}{q}},
$$

where $\mu(s)$ is Lebesgue measure of the set $S$, it is possible prove that

$$
\lim _{k_{2} \rightarrow \infty} \int_{t_{1}}^{t}\left[B\left(s, x^{\left(k_{2}\right)}(s)\right)-B\left(s, x_{2}(s)\right)\right]\left|u^{\left(k_{2}\right)}(s)\right| d s=0,
$$

and

$$
\int_{t_{1}}^{t} B\left(s, x^{\left(k_{2}\right)}(s)\right) u^{\left(k_{2}\right)}(s) d s \rightarrow \int_{t_{1}}^{t} B\left(s, x_{2}(s)\right) u^{*}(s) d s
$$


So, $x_{2}(t)=x_{1}\left(t_{1}\right)+I_{1}\left(x\left(t_{1}\right), v_{1}^{*}\right)+\varepsilon \int_{t_{1}}^{t}\left(A\left(s, x_{2}(s)\right)+B\left(s, x_{2}(s)\right) u^{*}(s)\right) d s$. Similar considerations are made at other intervals $\left[t_{k}, t_{k+1}\right]$. Hence, the function $x^{*}(t)$, constructed by formula (22), is the solution of the pulse system

$$
\begin{gathered}
x^{*}=\varepsilon\left[A\left(t, x^{*}\right)+B\left(t, x^{*}\right) u\right], \quad t \neq t_{i}, \quad i=1,2, \ldots, i\left(\frac{T}{\varepsilon}\right), \quad t \in\left[0, \frac{T}{\varepsilon}\right), \\
\left.\triangle x\right|_{t=t_{i}}=\varepsilon I_{i}\left(x^{*}\left(t_{i}\right), v_{i}^{*}\right), \quad i=1,2, \ldots, i\left(\frac{T}{\varepsilon}\right), \\
x\left(0, u(0), v_{i}^{*}\right)=x_{0}, \quad t_{i}<t_{i+1} .
\end{gathered}
$$

It remains to show that the control $\left(u^{*}, v^{*}\right)$ is optimal, i.e. that $J\left(u^{*}, v^{*}\right)=m$. We have

$$
m=\lim _{n \rightarrow \infty} J_{\varepsilon}\left(u^{(n)}, v^{(n)}\right)=\lim _{n \rightarrow \infty}\left[\varepsilon \int_{0}^{\frac{T}{\varepsilon}}\left(C\left(t, x^{(n)}(t)\right)+F\left(t, u^{(n)}(t)\right)\right) d t+\varepsilon \sum_{0<t_{i}<\frac{T}{\varepsilon}} \psi_{i}\left(x^{(n)}\left(t_{i}\right), v_{i}^{(n)}\right)\right] .
$$

From (20) by virtue of Lebesgue's theorem it follows that

$$
\lim _{n \rightarrow \infty} \varepsilon \int_{0}^{\frac{T}{\varepsilon}} C\left(t, x^{(n)}(t)\right) d t=\varepsilon \int_{0}^{\frac{T}{\varepsilon}} C\left(t, x^{*}(t)\right) d t .
$$

From the convexity by $u$ of the function $F(t, u)$ we also obtain that

$$
\lim _{n \rightarrow \infty} \varepsilon \int_{0}^{\frac{T}{\varepsilon}} F\left(t, u^{(n)}(t)\right) d t \geq \varepsilon \int_{0}^{\frac{T}{\varepsilon}} F\left(t, u^{*}(t)\right) d t
$$

It is also obvious that

$$
\lim _{n \rightarrow \infty} \varepsilon \sum_{0<t_{i}<\frac{T}{\varepsilon}} \psi_{i}\left(x^{(n)}\left(t_{i}\right), v_{i}^{(n)}\right)=\varepsilon \sum_{0<t_{i}<\frac{T}{\varepsilon}} \psi_{i}\left(x^{*}\left(t_{i}\right), v_{i}^{*}\right) .
$$

Therefore, $m=\lim _{n \rightarrow \infty} J\left(u^{(n)}, v^{(n)}\right) \geq J\left(u^{*}, v^{*}\right)=m$, which proves the existence of optimal control for each fixed $\varepsilon>0$.

Proving the existence of optimal control for an averaged system (4) is similar.

Let us prove the inequality (5). Note that the minimizing sequences and optimal controls for exact and averaged problems according to the inequality (15) satisfy the conditions of the lemma. Therefore, for controls $u, v$ from a given set and control $v_{0}$, chosen from condition a5), we have

$$
\begin{array}{r}
\left|J_{\varepsilon}(u, v)-\bar{J}\left(u, v_{0}\right)\right|=\mid \varepsilon \int_{0}^{\frac{T}{\varepsilon}}[C(t, x(t, u, v))+F(t, u)] d t+\sum_{0 \leq t_{i}<\frac{T}{\varepsilon}} \psi_{i}\left(x\left(t_{i}\right), v_{i}\right) \\
\quad-\varepsilon \int_{0}^{\frac{T}{\varepsilon}}\left[C_{0}\left(y\left(t, u, v_{0}\right)\right)+F(t, u)+\psi_{0}\left(y\left(t, u, v_{0}\right)\right)\right] d t \mid \\
\leq \varepsilon\left|\int_{0}^{\frac{T}{\varepsilon}}\left[C(t, x(t, u, v))-C_{0}\left(y\left(t, u, v_{0}\right)\right)\right] d t\right| \\
+\varepsilon\left|\int_{0}^{\frac{T}{\varepsilon}} \psi_{0}\left(y\left(t, u, v_{0}\right)\right) d t+\sum_{0 \leq t_{i}<\frac{T}{\varepsilon}} \psi_{i}\left(x\left(t_{i}\right), v_{i}\right)\right| .
\end{array}
$$


Similar to the terms estimates, made in the averaging lemma, we can show that the values of

$$
\varepsilon\left|\int_{0}^{\frac{T}{\varepsilon}}\left[C(t, x(t, u, v))-C_{0}\left(y\left(t, u, v_{0}\right)\right)\right] d t\right|
$$

and

$$
\varepsilon\left|\int_{0}^{\frac{T}{\varepsilon}} \psi_{0}\left(y\left(t, u, v_{0}\right)\right) d t+\sum_{0 \leq t_{i}<\frac{T}{\varepsilon}} \psi_{i}\left(x\left(t_{i}\right), v_{i}\right)\right|
$$

from (24) can be made arbitrarily small by choosing a sufficiently small $\varepsilon$.

Therefore, for an arbitrary $\eta>0$ there exists $\varepsilon_{0}>0$ such that for $\varepsilon<\varepsilon_{0}$ :

$$
\left|J_{\varepsilon}(u, v)-\bar{J}_{\mathcal{E}}\left(u, v_{0}\right)\right|<\eta
$$

Let $\left(u_{0}^{*}(\varepsilon), v_{0}^{*}(\varepsilon)\right)$ be the optimal pair for the averaged problem (4), and let $\left(u^{*}(\varepsilon), v^{*}(\varepsilon)\right)$ be the optimal pair for the original problem (1). Then

$$
J_{\varepsilon}^{*} \leq J_{\varepsilon}\left(u_{0}^{*}(\varepsilon), v_{0}^{*}(\varepsilon)\right)=\bar{J}_{\varepsilon}^{*}+J_{\varepsilon}\left(u_{0}^{*}(\varepsilon), v_{0}^{*}(\varepsilon)\right)-\bar{J}_{\mathcal{\varepsilon}}\left(u_{0}^{*}(\varepsilon), v_{0}^{*}(\varepsilon)\right) .
$$

But for an arbitrary $\eta_{1}>0$ for sufficiently small $\varepsilon>0$, by virtue of (25) we have:

$$
\left|J_{\varepsilon}\left(u_{0}^{*}(\varepsilon), v_{0}^{*}(\varepsilon)\right)-\bar{J}_{\mathcal{\varepsilon}}\left(u_{0}^{*}(\varepsilon), v_{0}^{*}(\varepsilon)\right)\right| \leq \eta_{1},
$$

then

$$
J_{\mathcal{E}}^{*}-\bar{J}_{\mathcal{E}}^{*} \leq \eta_{1}
$$

Since the pair $\left(u^{*}(\varepsilon), v^{*}(\varepsilon)\right)$ is admissible, there is an admissible pair $\left(u^{*}(\varepsilon), v_{0}(\varepsilon)\right)$ from a5). So, $\bar{J}_{\varepsilon}^{*}=\bar{J}_{\varepsilon}\left(u_{0}^{*}(\varepsilon), v_{0}^{*}(\varepsilon)\right) \leq \bar{J}_{\varepsilon}\left(u^{*}, v_{0}\right)+J_{\varepsilon}^{*}-J_{\varepsilon}\left(u^{*}(\varepsilon), v^{*}(\varepsilon)\right)$.

But by virtue of (25) for sufficiently small $\varepsilon$ the estimate

$$
\left|\bar{J}_{\varepsilon}\left(u^{*}(\varepsilon), v_{0}(\varepsilon)\right)-J_{\varepsilon}\left(u^{*}(\varepsilon), v^{*}(\varepsilon)\right)\right| \leq \eta_{1}
$$

holds.

So, from the inequality

$$
\bar{J}_{\varepsilon}^{*} \leq J_{\varepsilon}^{*}+\eta_{1}
$$

and from (26) we obtain

$$
\left|\bar{J}_{\varepsilon}^{*}-J_{\varepsilon}^{*}\right| \leq \eta_{1}
$$

Now, we have the estimate

$$
\left|J_{\mathcal{\varepsilon}}\left(u_{0}^{*}(\varepsilon), v_{0}^{*}(\varepsilon)\right)-J_{\mathcal{\varepsilon}}^{*}\right| \leq\left|J_{\mathcal{\varepsilon}}\left(u_{0}^{*}(\varepsilon), v_{0}^{*}(\varepsilon)\right)-\bar{J}_{\mathcal{\varepsilon}}^{*}\right|+\left|\bar{J}_{\mathcal{\varepsilon}}^{*}-J_{\mathcal{\varepsilon}}^{*}\right| .
$$

The first term in (28) according to (25) does not exceed $\eta_{1}$ for sufficiently small $\varepsilon$.

Due to the arbitrariness of $\eta_{1}$ from (27) and (28) we obtain the proximity of the optimal controls of the initial problem (1) and the averaged problem (4).

Thus, the theorem is proved. 


\section{REFERENCES}

[1] Kovalchuk T.V., Mogilova V.V., Shovkoplyas T.V. The averaging method in the problems of optimal control by impulsive systems. J. Math. Sci. 2020, 247 (2), 314-327. doi:10.1007/s10958-020-04804-2

[2] Kravets' V.I., Koval'chuk T.V., Mohyl'ova V.V., Stanzhyts'kyi O.M. Application of the Method of Averaging to the Problems of Optimal control Over Functional-Differential Equations. Ukrainian Math. J. 2018, 70 (2), $232-242$. doi:10.1007/s11253-018-1497-9

[3] Nosenko T.V., Stanzhytskyi O.M. The avergaging method in some optimal control problems. Nonlinear Oscill. 2008, 11 (4), 512-519. doi:10.1007/s11072-009-0049-5

[4] Samoilenko A.M. Averaging method in systems with tremors. Mathematician. Physics 1971, 9, 101-117. (in Russian)

[5] Samoilenko A.M., Perestyuk M.O. Impulsive differential equations. World Scientific, Singapore-NewJersey-London-Hong-Kong, 1995.

[6] Samoilenko A.M., Stanzhitskii A.N. On the averaging of differential equations on an infinite interval. Diff. Equat. 2006, 42 (4), 505-511. doi:10.1134/S0012266106040070

[7] Stanzhitskii A.M. Investigation of Invariant Sets of Itô Stochastic Systems with the Use of Lyapunov Functions. Ukrainian Math. J. 2001, 53 (2), 323-327. doi:10.1023/ A:1010437625118

[8] Stanzhitskii A.N., Dobrodzii T.V. Study of optimal control problems on the half-line by the avergaging method. Diff. Equat. 2011, 47 (2), 264-277. doi:10.1134/S0012266111020121

[9] Plotnikov V.A. Averaging method in control problems. Lybid, Kiev, Odessa, 1992. (in Russian)

[10] Yosida K. Functional Analysis. Mir, Moscow, 1967. (in Russian)

Received 15.09.2020

Ковальчук Т.В., Могильова В.В., Станжицький О.М., Шовкопляс Т.В. Застосування методу усереднення до задаи оптимального керування імпульсними системами // Карпатські матем. публ. — 2020. — T.12, №2. — C. 504-521.

Розглянуто задачу оптимального керування на скінченному часовому інтервалі для системи диференціальних рівнянь з імпульсною дією у фіксовані моменти часу та відповідну ӥй усереднену систему звичайних диференціальних рівнянь.

$\triangle$ оведено існування оптимального керування точної та усередненої задач, а також встановлено, що оптимальне керування усередненої задачі здійснює наближений оптимальний синтез точної задачі. Основним результатом роботи є теорема, яка доводить, що оптимальне керування усередненої задачі $є$ майже оптимальним для точної. Отримано обгрунтування близькості розв'язків точної та усередненої задач.

Ключові слова $і$ фрази: задача оптимального керування, оптимальне керування, точна задача, усереднена задача, допустиме керування, умови усереднення, слабка збіжність, компактність, напівнеперервність, критерій якості, мінімізуюча послідовність. 